\title{
LA NAVEGACIÓN EN EL ESTUARIO DE GUADALQUIVIR. DIMENSIONES ECOLÓGICAS, ECONÓMICAS, SOCIALES, JURÍDICAS Y POLÍTICAS
}

\author{
LEANDRO DEL MORAL ITUARTE \\ lmoral@us.es \\ UNIVERSIDAD DE SEVILLA \\ http://dx.doi.org/10.25267/Riparia_sup.2018.i1.03
}

\section{El mito de la navegación en la Antigüedad}

Durante siglos las informaciones sobre la navegación del Guadalquivir hasta Córdoba en la Antigüedad, debidas especialmente a Estrabón y a Plinio el Viejo aparecen como un punto de referencia permanente en la Baja Andalucía. Desde el siglo XIV en adelante, la discusión y los planes sobre el río tienen presente, como telón de fondo, ese panorama de la edad de oro de la navegación que los tiempos clásicos supusieron. Sin embargo, al margen de las mejores condiciones de navegación que indudablemente tenía el estuario - en una fase de colmatación menos avanzada pocas son las noticias que en realidad tenemos sobre la navegación propiamente fluvial en aquella época. Está claro, en primer lugar, que ya en la antigüedad la navegación marítima llegaba solamente a Sevilla (Hispalis) y que únicamente se podía remontar el río hasta el final del estuario, en Alcalá del Río (Ilipa Magna), con barcos de menor calado. A partir de este punto sólo se podían utilizar embarcaciones pequeñas, "lyntres" o barcas de río como las llama Estrabón. Se trataba en definitiva de un transporte fraccionado en barcos de mayor a menor calado según se alejara del estuario, que constituía la comunicación natural entre dos núcleos principales, Itálica y Córdoba, y su conexión marítima a partir de Sevilla.

Se discute entre los especialistas las condiciones que hacían posible esta limitada navegabilidad del río. De una parte, la presumible mayor abundancia y regularidad del caudal; de otra, el nivel e influencia de la intervención humana sobre el cauce, temas sobre los que las opiniones están muy enfrentadas. George Bonsor consideró en su ya clásico estudio, que debía existir todo un sistema de diques y esclusas ("river dams and locks") para regular el régimen del río, contener sus crecidas y aliviar sus épocas de escasez. Los diques principales, según esta hipótesis, se reconocerían en los restos conservados en Alcalá del Río y Cantillana (Naeva), primeros de una serie que se extendería río arriba coincidiendo en su emplazamiento con el topónimo "portus". Otros autores, como Thouvenot, descartan tal interpretación pero, desde luego, reconocen que el mantenimiento del carácter navegable del río requeriría un estudio paciente de su régimen al mismo tiempo que una gran cantidad de cuidados y de obras, especialmente de rectificación del cauce. En ese sentido, hay constancia de la existencia de una procuratela ("procurator ad ripam Baetis") con la misión específica del cuidado del río en todo lo concerniente a la conservación de sus riberas y al mantenimiento de los puertos. Otro aspecto muy interesante es la existencia de una legislación dirigida a garantizar los caudales fluviales de estiaje para la navegación, frente a su eventual derivación hacia otros usos. Es este un antecedente remoto de una normativa de aguas que muchos siglos después volverá a repetirse con importantes repercusiones para el sistema hidráulico de la cuenca baja del Guadalquivir. 
Lo cierto es que han llegado hasta nosotros restos de potentes construcciones localizadas en lugares que aún hoy siguen siendo claves en la red de la obra hidráulica. Destaca, además de los mencionados, el dique llamado el Higuerón, en Peñaflor (Celti), inmediatamente después de la confluencia del Genil cerca de donde en la actualidad se emplaza la presa de derivación del canal del Bajo Guadalquivir. Otro de importancia se sitúa en Coria del Río (Axati), que se adentra en el río desde la margen derecha en dirección opuesta a la de la corriente, con unas dimensiones de unos 50 metros de longitud por unos 4 de anchura. Este dique transversal imprimía una dirección fija a las aguas, evitando remolinos y contribuyendo a asegurar una corriente uniforme y un caudal constante en el thalweg. Este tipo de actuaciones con diques transversales quizá sean las que mejor demuestren la gran pericia de los ingenieros romanos para hacer frente a uno de los mayores problemas que presenta el cauce del Guadalquivir. Efectivamente, la inestabilidad de las márgenes que entre otros problemas dificultaba el mantenimiento de los caminos de sirga, la excesiva anchura del cauce, con su repercusión sobre la altura de las aguas y la aparición de islas y bifurcaciones, fueron problemas que, según algunas interpretaciones, la ingeniería romana supo manejar con eficacia.

En cualquier caso, y es la imagen que transcendió a la posteridad, el Guadalquivir constituía el eje en torno al que, a partir del mandato de Augusto, se produjo la explosión económica de la Bética, a cuyo calor los productos de su minería y agricultura alcanzaron todos los extremos del Mediterráneo y las costas atlánticas del Imperio.

\section{Los términos del debate actual}

Con ese remoto telón de fondo, durante años políticos municipales, sindicalistas y empresarios bombardean a la opinión pública con el mensaje de que la supervivencia del puerto de Sevilla se juega en la realización del dragado de profundización del estuario del Guadalquivir. Renace así el discurso que se oye en Sevilla cada dos o tres décadas desde hace siglos: el puerto agoniza y si se acomete una gran operación infraestructural (en la actualidad un dragado de profundización) no solo se afrontará esta amenaza de colapso sino que se relanzará la actividad del puerto a niveles muy superiores.

¿Cuál es la realidad? El 2 de octubre de 2003 se publicó la Declaración de Impacto Ambiental (DIA) del Proyecto "Actuaciones de mejora en los accesos marítimos al puerto de Sevilla". En cumplimiento de lo determinado por la DIA se constituyó una Comisión Científica que tras varias reuniones con la Autoridad Portuaria acordó encargar el Estudio "Propuesta Metodológica para diagnosticar y pronosticar las consecuencias de las actuaciones humanas en el estuario del Guadalquivir" a un equipo investigador para cubrir las lagunas de información detectadas en el proyecto. Con fecha 14 de octubre de 2010, se reunió en Sevilla la Comisión Científica, junto al equipo investigador responsable del Estudio, representantes del Puerto de Sevilla, funcionarios de las administraciones y observadores. Como resultado de ello, la Comisión de expertos redactó un dictamen el 12 de noviembre de 2010 que entre otras cosas dice: "La situación actual (del estuario) requiere la intervención rápida y coordinada de las administraciones implicadas en la planificación y gestión del estuario, de la cuenca del Guadalquivir y de la costa, de modo que se revierta la tendencia actual que lleva al colapso del estuario y a la costa que de él depende [...] el conocimiento actual del estuario confirma que la profundización del dragado del Guadalquivir no es el único proyecto que debe preocupar para el futuro del estuario, ya que la modernización de regadíos del arrozal, la urbanización y construcción de campos de golf en zonas de llanos mareales o los resguardos en bonanza, tendrían 
igualmente efectos negativos y ninguno positivo para el estuario, Doñana y la costa". A continuación se dice: “...recomendamos que se desestime definitivamente el dragado de profundización y que de mantener la autoridad portuaria de Sevilla su voluntad de realizar dicho dragado, inicie un nuevo procedimiento de EIA una vez las condiciones del estuario hayan mejorado".

\section{E1 Plan Hidrológico de la cuenca, UNESCO, Comisión Europea y Tribunal Supremo}

En 2010 la Confederación Hidrográfica del Guadalquivir presentó la Propuesta de Plan Hidrológico de la Demarcación del Guadalquivir, sin que en tal documento fundamental se hiciera referencia alguna al dragado del estuario. Dos años más tarde se aprobó la versión final de este Plan, en el que se incluye el dragado de profundización, sin hacer mención a los nuevos conocimientos adquiridos sobre el estuario y a las recomendaciones hechas por los científicos y la Comisión creada al efecto.

En junio de 2013 en la 37 Sesión del Comité de Patrimonio Mundial de la UNESCO celebrada en Phnom Penh, se aprueba: "Urgir al gobierno de España a no permitir ninguna profundización del dragado en el Bajo Guadalquivir, en línea con las recomendaciones de la Comisión Científica y con anteriores decisiones de la Unesco, y a integrar las conclusiones de la Comisión Científica como condiciones vinculantes en la Declaración de Impacto Ambiental...”.

El 12 de octubre de este mismo año la Comisión Europea abre un procedimiento de infracción contra España por el proyecto de dragado a raíz de la queja interpuesta por WWF España. Las autoridades europeas confirman así que el proyecto de la Autoridad Portuaria de Sevilla puede vulnerar la normativa comunitaria de protección de la naturaleza, por los graves impactos que ocasionaría sobre Doñana y otros espacios de la Red Natura 2000.

En febrero de 2015 el Tribunal Supremo (TS) dicta una sentencia referida al recurso de WWF contra el Real Decreto de mayo de 2013 que aprobó el Plan Hidrológico del Guadalquivir (PHG). En esta sentencia el TS declaró nula la incorporación del proyecto de dragado del estuario en ese Plan. Como señala la sentencia, toda actuación sobre el dominio público hidráulico se encuentra sujeta a la planificación hidrológica, pues los planes proyectan y concretan, sobre el territorio de una demarcación hidrográfica específica, las previsiones generales de la Ley. Por esto, cualquier intento de obviar esta sentencia, argumentando que se refiere al Plan Hidrológico y no al proyecto de dragado de profundización, está destinado al fracaso.

¿Qué había hecho la Confederación Hidrográfica $(\mathrm{CHG})$ para que el TS declaré nulo, en este punto, su Plan? En primer lugar, la Directiva Marco del Agua (DMA) manda conservar o alcanzar el buen estado ecológico de las masas de agua (ríos, estuarios, deltas, lagos, acuíferos, aguas litorales) en el año 2015. La DMA prevé la posibilidad de aplicar excepciones: reconocer que algunas masas de agua ya están muy modificadas y no podrán alcanzar el objetivo de "buen estado ecológico", sino uno más modesto, el "buen potencial ecológico"; retrasar la meta al año 2021 o 2027; establecer objetivos menos rigurosos; incluso aceptar nuevas modificaciones que acarreen deterioros adicionales (art. 4 de la DMA). Pero todo esto, como establece específicamente el Reglamento de la planificación hidrológica, con rigor, demostrando que los motivos de los deterioros son de interés público superior y que la pérdida de los beneficios para la sociedad se compensan por los beneficios de las nuevas modificaciones para la salud pública, el mantenimiento de la seguridad humana o el desarrollo sostenible (art. 39 del Reglamento). 
Nada de esto hace la CHG. Por el contrario, utilizando exclusivamente datos de un estudio financiado por el Puerto hace quince años (pág. 91 y siguientes de la Memoria, 2013), muy desprestigiados en círculos académicos y científicos, el PHG incluye una medida, el dragado, que va a suponer fuertes alteraciones de las masas de aguas del estuario, con deterioro de su estado actual. Baste con citar, como recuerda la sentencia, la conclusión del dictamen de la Comisión Científica de noviembre de 2010: "El dragado de profundización no es recomendable en la situación actual, por repercutir negativamente en la dinámica, morfología y biodiversidad del estuario y por lo tanto de Doñana, por no mejorar la situación frente a procesos extremos (turbidez y salinidad) y limitar severamente la capacidad de minimizar su duración y sus efectos. (...) En una situación diferente, una vez se haya puesto en marcha una gestión integral del estuario y se acometan actuaciones que permitan, entre otros, recuperar llanuras mareales, reconectar los brazos del río con el cauce principal, aumentar y mejorar los aportes de caudales de agua dulce, reducir el aporte de sedimentos, nutrientes y pesticidas desde la cuenca del Guadalquivir, y una vez que se haya comprobado que el estuario ha mejorado su funcionalidad, podrá volver a evaluarse la posibilidad de un dragado de profundización en el estuario".

Frente a esta conclusión contundente, el PHG se limitaba a señalar, genéricamente, la importancia del trasporte marítimo y la navegación, atendiendo a su actividad económica, informando que Sevilla es el único puerto fluvial de España. Pero el Plan, señala la sentencia, ha de justificar "específicamente" las actuaciones que comporten un deterioro de las masas de agua y, por lo tanto, se aparten de la regla general que exige no empeorar el estado de las aguas.

En segundo lugar, la realización del dragado del canal del Puerto de Sevilla no puede tener, por su propia naturaleza, el carácter de medida complementaria para la conservación o recuperación de la buena calidad del agua, como la presenta el PHG. De acuerdo con la sentencia, el dragado es una actuación que puede modificar las masas de agua y que precisa él mismo del correspondiente programa de medidas que tenga en cuenta los estudios realizados.

En marzo de 2015 la Comisión Europea emite un Dictamen Motivado en el que se pide que España sea prudente al mejorar el acceso marítimo al puerto de Sevilla y evalúe las posibles repercusiones negativas en espacios de la red Natura 2000, tales como el Parque Nacional de Doñana. El Dictamen señala que un reciente estudio científico encargado por las autoridades españolas indica que el dragado del canal de navegación tendría graves consecuencias para la forma y la biodiversidad del estuario, que repercutirían en los cercanos espacios naturales protegidos. De conformidad con la Directiva sobre los hábitats, cualquier plan o programa que pueda afectar de manera significativa a un espacio protegido debe ser objeto de una "evaluación apropiada" de sus repercusiones. La Directiva permite mantener un proyecto, por razones imperiosas de interés público, a pesar de una evaluación negativa, pero entonces se necesitan medidas compensatorias para garantizar que la coherencia global de la red Natura 2000 quede protegida. España no ha estudiado detalladamente las repercusiones del proyecto ni las soluciones alternativas, lo que es un requisito básico a tenor de la Directiva, concluye el Dictamen.

Aunque los grupos de presión que apoyan el proyecto no desisten del empeño, los argumentos científicos y legales respaldan a los sectores sociales que defienden una estrategia alternativa para este importante sistema territorial de Andalucía.

\section{Evolución posterior del tema y perspectivas de futuro}

Aunque el PHG se aprobó en 2013, el documento correspondía al ciclo 2009-2015 y ya en este último año tocaba su renovación, al comenzar el segundo ciclo de 
planificación hidrológica 2015-2021 establecido por la DMA. En el nuevo Plan, la CHG volvió a incluir el dragado de profundización del estuario, con los mismos argumentos, aunque tratando de evitar algunos de los errores de bulto que cometía en el anterior documento. Se reconoce que el dragado no es una medida complementaria para mejorar el estado de las masas de agua afectadas, sino una intervención que produce deterioros añadidos, a la que se le tienen que aplicar todas las cautelas y justificaciones (ecológicas, técnicas, económicas y sociales) que este tipo de actuaciones exigen. En este contexto se sitúa la actual campaña de legitimación científico-técnica de la estrategia portuaria.

Mientras tanto, la Autoridad Portuaria, que afirmaba insistentemente que el dinero europeo disponible para el dragado ( 35 millones de euros) se perdería si este no se ejecutaba en 2015, ya aplica estos fondos, sin ningún problema, a la mejora de las conexiones viarias y ferroviarias del puerto.

Hasta aquí la larga y compleja tramitación administrativa del proyecto y sus derivadas a escala nacional e internacional, pero el problema trasciende con mucho de una mera cuestión jurídica. Tampoco se trata exclusivamente de una amenaza a Doñana, ni menos aún a una Doñana concebida en sus estrictos límites administrativos de espacio protegido e inventario de especies. Se trata, en todo caso, de una amenaza a un proyecto, Doñana, que significa laboratorio de procesos, ideas y experiencias de desarrollo sostenible. Además de los impactos ambientales, el proyecto tiene graves afecciones sobre otras actividades productivas (otros puertos andaluces, otras zonas industriales) e ignora la existencia de otros agentes en conflicto como arroceros, piscicultores, pescadores y marisqueo, salineros, y proyectos de desarrollo rural y turístico de los Ayuntamientos ribereños. El estudio económico de los efectos inducidos por la ampliación del Puerto es antiguo, controvertido, hecho a la carta y extremadamente débil.

¿Cuál es el nuevo enfoque que el importante tema del puerto de Sevilla requiere? Pasar de una perspectiva centrada exclusivamente en los intereses sectoriales y actuales del puerto a una perspectiva integradora de la complejidad de funciones, actividades económicas, sociales y ecológicas presentes en el estuario. Frente a una perspectiva coyuntural, construir una perspectiva que tenga en cuenta la experiencia del largo proceso de intervención sobre el estuario. Frente a una perspectiva de lucha contra la naturaleza, de intervención quirúrgica y artificialización continua, diseñar una estrategia de adaptación y negociación con la naturaleza. Frente a la opacidad y la ausencia de rendición de cuentas de los centros de poder actuales, garantizar la transparencia y admitir la participación en el debate con las opiniones contrarias. 


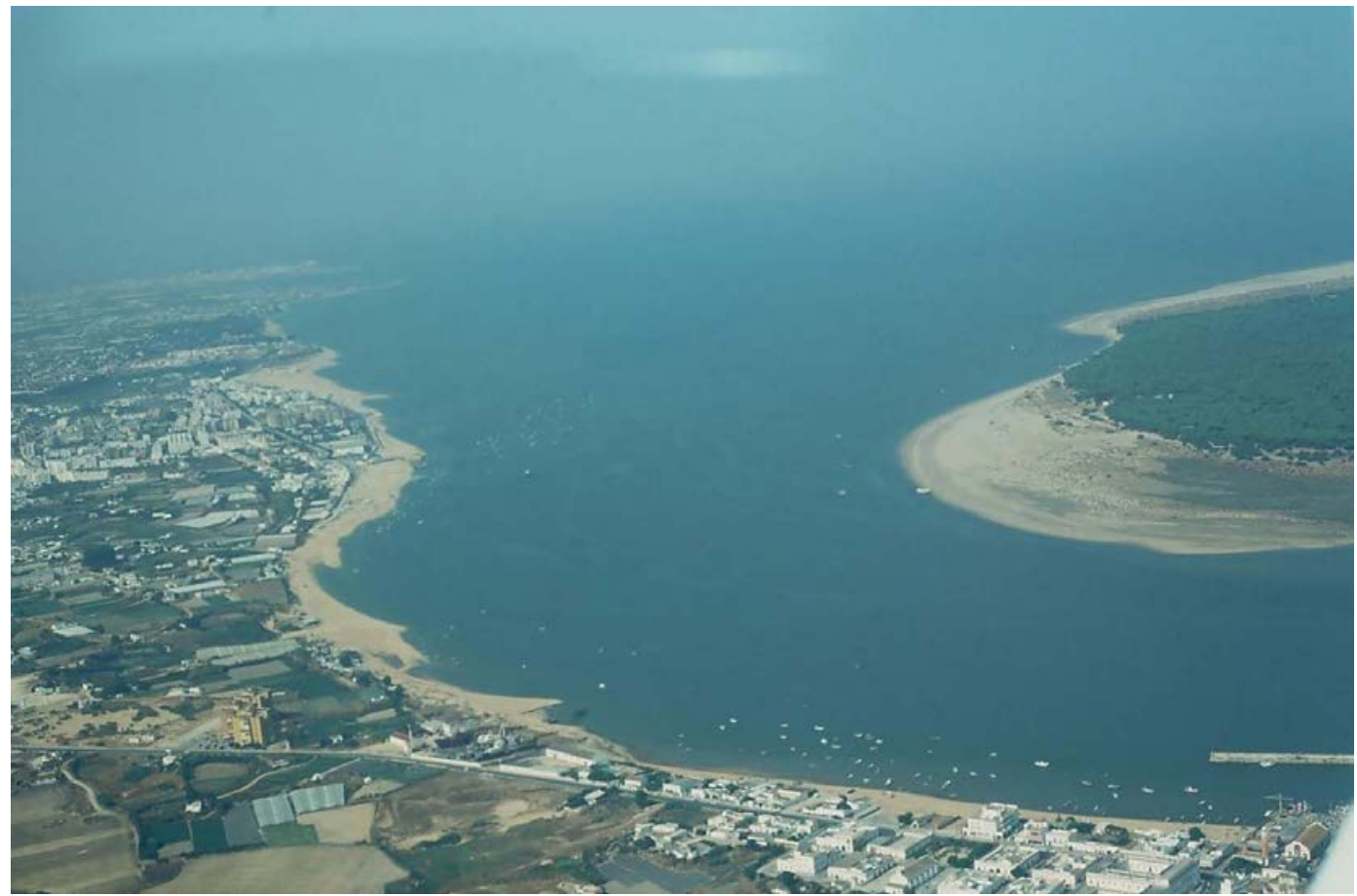

Desembocadura del río Guadalquivir en Sanlúcar de Barrameda (foto: L. DEL MORAL, 1991).

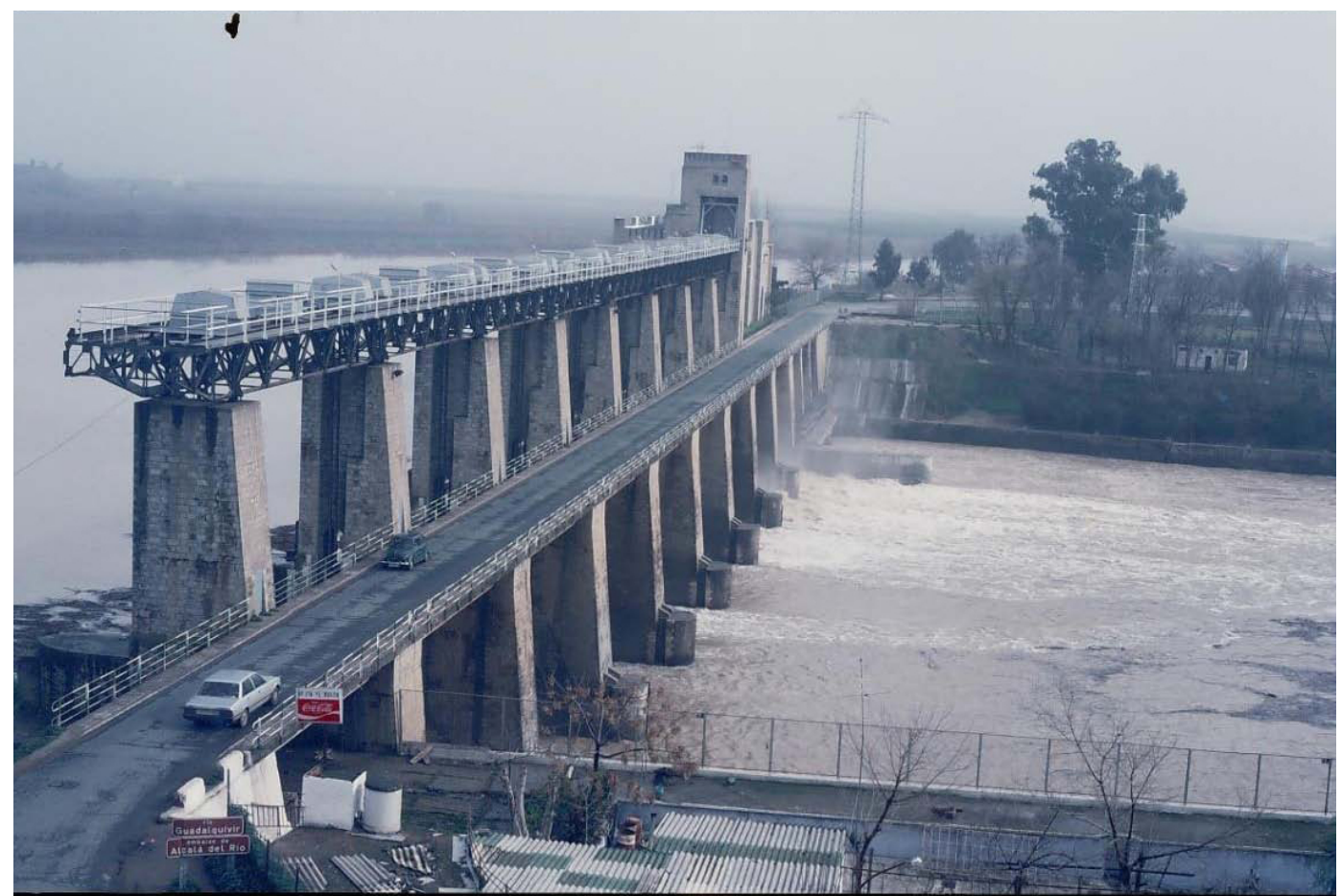

Alcalá del Río, la antigua llipa Magna, el final del estuario del Guadalquivir (foto: L. DEL MORAL, 1991). 


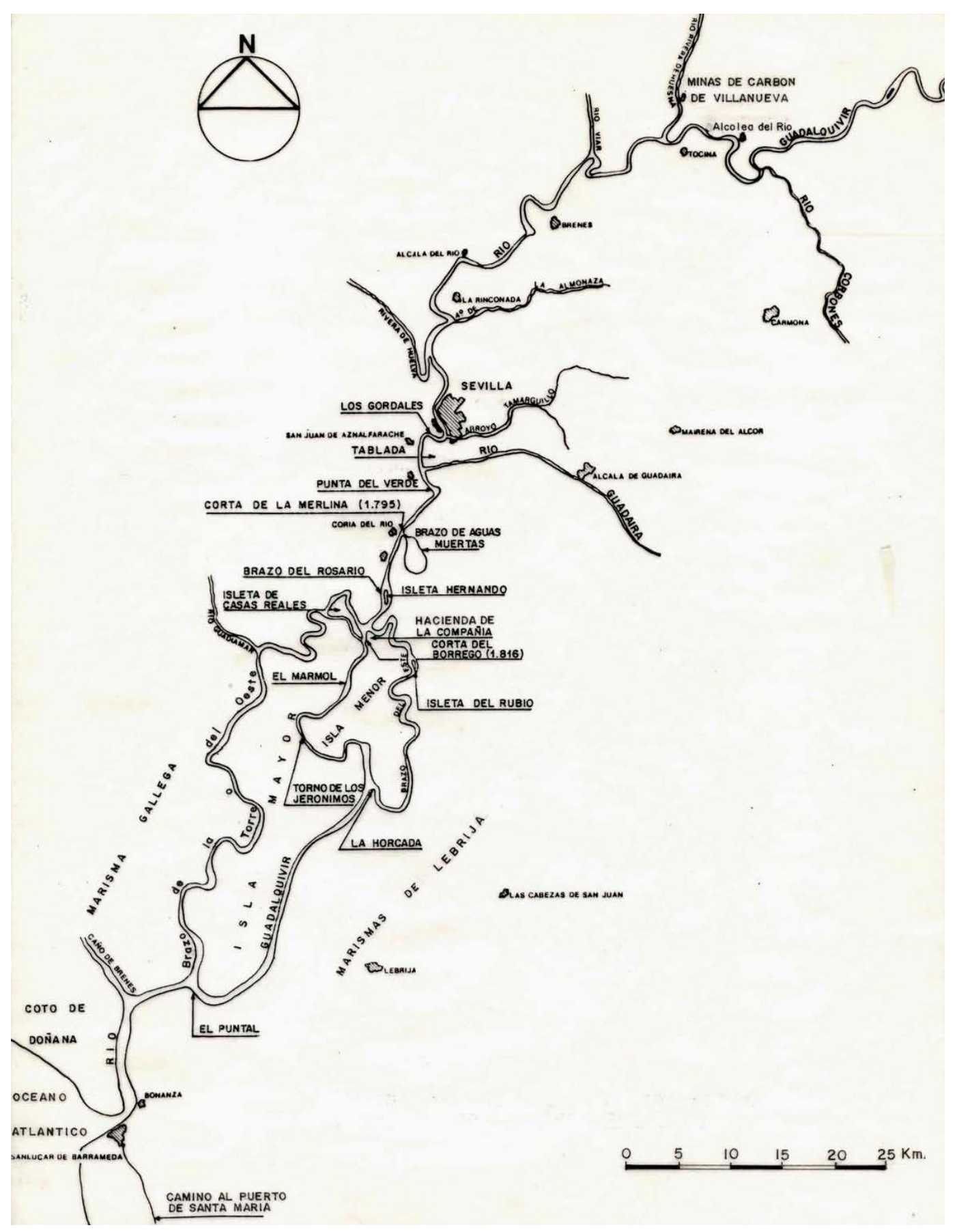

El estuario de la Compaña de Navegación del Guadalquivir (fuente L. DEL MORAL, 1990). 


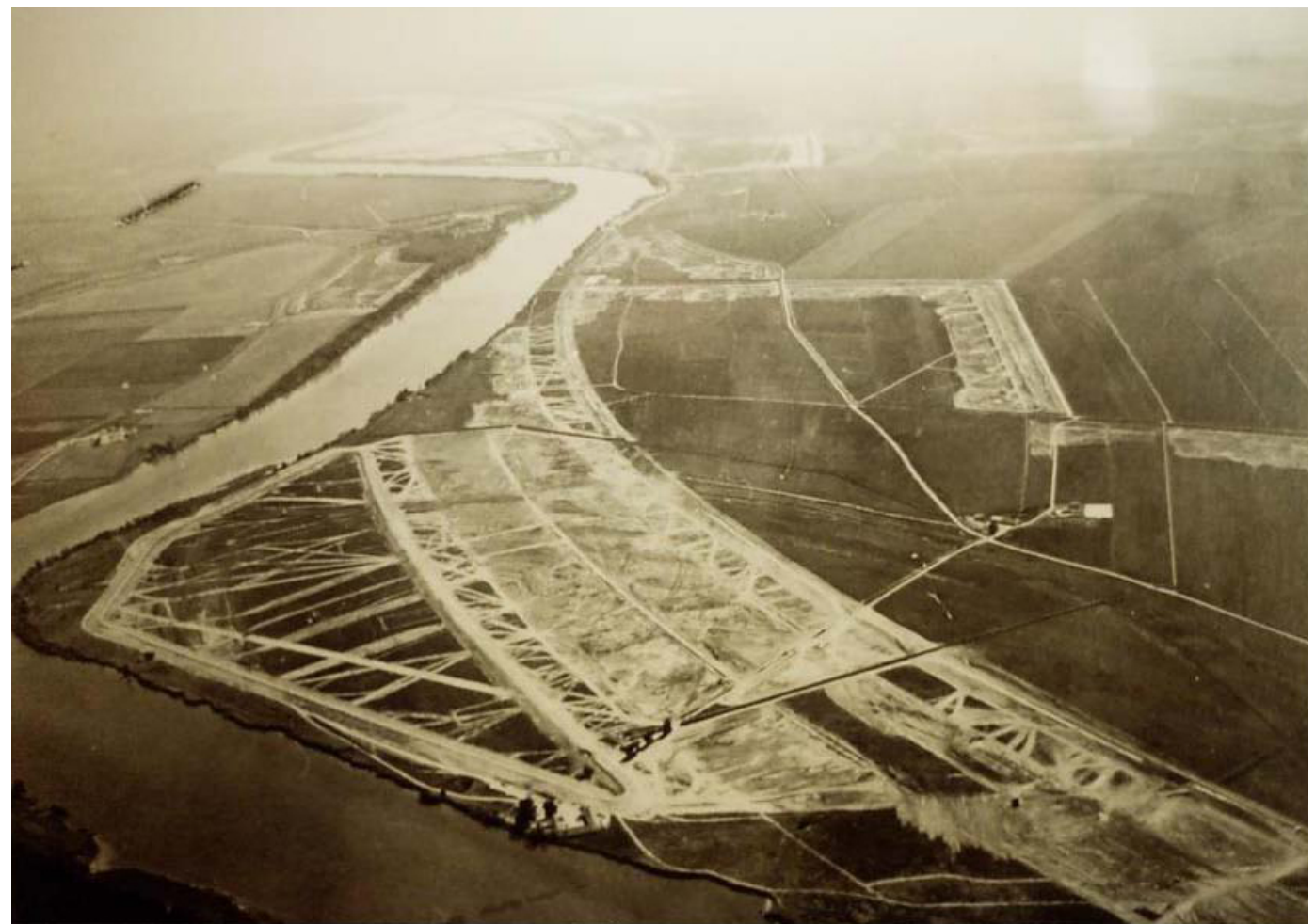

Las cortas de la Isletay de los Olivillos, 1968 (fuente L. DEL MORAL, 1990). 